\title{
In-situ TEM Characterization of Ultra-robust Memristors Based on Fully Layered Two-dimensional Materials
}

\author{
Songhua Cai ${ }^{1}$, Miao Wang ${ }^{2}$, Xiaoqing Pan ${ }^{1,3}$, J. Joshua Yang $^{4}$, Feng Miao ${ }^{2}$, Peng Wang ${ }^{1}$
}

1. National Laboratory of Solid State Microstructures, College of Engineering and Applied Sciences and Collaborative Innovation Center of Advanced Microstructures, Nanjing University, Nanjing, China.

2. School of Physics, Collaborative Innovation Center of Advanced Microstructures, Nanjing University, Nanjing, China.

3. Department of Chemical Engineering and Materials Science and Department of Physics and Astronomy, University of California, Irvine, CA, USA.

4. Department of Electrical and Computer Engineering, University of Massachusetts, Amherst, MA, USA.

Memristor is a promising candidate for storage and neuromorphic electronics in the future [1] because of advantages such as device scalability, multi-state storage, high switching speed and endurance, as well as CMOS compatibility [2]. While the reliability of memristors in extreme environments such as high temperature and bending substrates has not been much studied yet, we designed and fabricated an ultrarobust memristor consists of a Van der Waals ( $\mathrm{vdW}$ ) heterostructure laminated with graphene/ $\mathrm{MoS}_{2-}$ ${ }_{\mathrm{x}} \mathrm{O}_{\mathrm{x}} /$ graphene, all fully layered $2 \mathrm{D}$ materials. Our test suggests a total life up to $10^{7}$ cycles and ultra-high thermal stability that can work properly even temperature up to $340^{\circ} \mathrm{C}$ [3]. These results show that vdW heterostructures made up through stacked 2D layered materials can integrate each component's unique properties then fit actual requirements in detection and production.

To investigate the temperature stability of the heterostructural device, we carried out a TEM in-situ heating test to same $\mathrm{MoS}_{2-\mathrm{x}} \mathrm{O}_{\mathrm{x}}$ used in our devices. $\mathrm{MoS}_{2-\mathrm{x}} \mathrm{O}_{\mathrm{x}}$ nanosheets were prepared by ultrasonic exfoliation then dropped onto a DENSsolution in-situ heating chip. Before in-situ experiment, sample was heated to $160^{\circ} \mathrm{C}$ in atmosphere and kept 90 mins for thermal oxidation. In-situ heating results prove that layered $\mathrm{MoS}_{2-\mathrm{x}} \mathrm{O}_{\mathrm{x}}$ can still retain undamaged up to $800^{\circ} \mathrm{C}$, exhibits excellent thermal stability. From HRTEM images and FFT results, the lattice constant of $\mathrm{MoS}_{2-\mathrm{x}} \mathrm{O}_{\mathrm{x}}$ was measured to be $3.16 \AA$ at room temperature, which matches former reported values [4]. At $800^{\circ} \mathrm{C}$, the actual lattice constant slightly increased to $3.19 \AA$, indicates a tiny lattice thermal expansion and expansion ratio is about $1 \%$.

Furthermore, we carried out in situ scanning transmission electron microscopy (STEM) investigations to investigate the working mechanism of GMG memristor. In-situ device sample was made by FEI helios 600i dual-beam FIB system and fabricated to our home made in-situ electrical testing chip. HAADF and STEM-EDS analyses were used to reveal structure change and mass migration during switching process. The STEM and HRTEM images were obtained on a FEI Titan Cubed G2 60-300 aberration corrected $\mathrm{S} / \mathrm{TEM}$. The operation voltage of $60 \mathrm{kV}$ was used to reduce electron beam damage to graphene and $\mathrm{MoS}_{2-}$ ${ }_{x} \mathrm{O}_{x}$. EDS analyses were carried out using Bruker SuperEDX four-detector system. By switching the device to $\mathrm{ON} / \mathrm{OFF}$ states inside the $\mathrm{S} / \mathrm{TEM}$, we were able to observe the structural changing area of the conduction channel in $\mathrm{MoS}_{2-\mathrm{x}} \mathrm{O}_{\mathrm{x}}$ layer in real-time and attributed the switching mechanism to the migration of oxygen ions. The numbers of Mo, $\mathrm{S}$ and $\mathrm{O}$ were measured and normalized as atom percentage. $\mathrm{MoS}_{2-}$ ${ }_{\mathrm{x}} \mathrm{O}_{\mathrm{x}}$ layer in pristine state shows a normal atom distribution with $\mathrm{Mo}:(\mathrm{S}+\mathrm{O}) \approx 1: 2$. Then at $\mathrm{ON}$ state a reduction of $\mathrm{S}$ and $\mathrm{O}$ atoms was observed in conduction channel region, where Mo : $(\mathrm{S}+\mathrm{O})$ is about 1 : 1.2. At $\mathrm{OFF}$ state, an increase of $\mathrm{O}$ atoms was observed in conduction channel region, led to ratio of Mo 
$:(\mathrm{S}+\mathrm{O})$ back to near $1: 2$. As a result, the changing of oxygen vacancy intensity caused two different resistance states.

References:

[1] L Chua, IEEE Trans. Circuit Theory 18 (1971), p. 507.

[2] J Yang, D Strukov and D Stewart, Nat. Nanotechnol. 8 (2013), p. 13.

[3] M Wang, et al, Nature Electronic (2018), https://doi.org/10.1038/s41928-018-0021-4.

[4] S Helveg, et al, Phys. Rev. Lett. 84 (2000), p. 5.

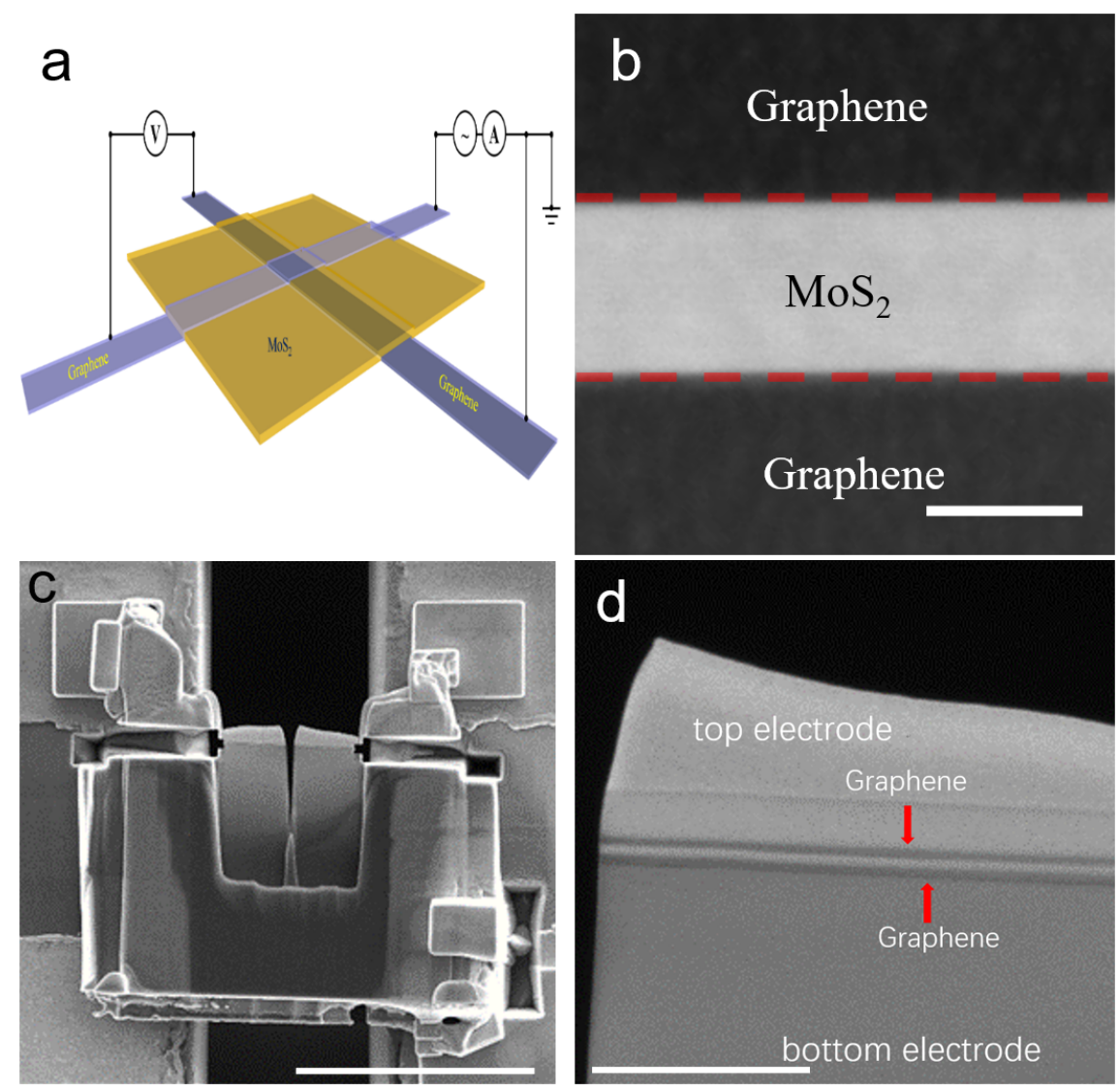

Figure 1. (a) schematic drawing of the GMG devices. (b) Cross-section HAADF image of a pristine GMG device. Scale bar: $20 \mathrm{~nm}$. (c) in-situ GMG device sample welded on home-made in-situ electrical testing chip by dual-beam FIB system. Scale bar: $10 \mu \mathrm{m}$. (d) high magnification SEM image of in-situ cross section sample, shows a sandwich structure. Scale bar: $1 \mu \mathrm{m}$. 